\title{
Topographische Daten aus Laserscanning als Grundlage für Hydrologie und Wasserwirtschaft
}

Zusammenfassung: In den letzten 15 Jahren hat Airborne Laserscanning (ALS) die Geländeaufnahme durch bislang unerreichte Datendichte von mehreren Punkten $/ \mathrm{m}^{2}$ sowie Höhengenauigkeit von besser als $15 \mathrm{~cm}$ revolutioniert. Als präzise geometrische Datengrundlage für Gefahrenzonenplanung, Ausweisung von Überschwemmungsflächen, aber auch für wasserbiologische Fragestellungen sind topographische Daten aus Laserscanning heute nicht mehr wegzudenken.

Der vorliegende Artikel gibt einen Überblick über das Einsatzspektrum von ALSDaten in der wasserwirtschaftlichen Praxis. Im ersten Teil wird der Aufbau eines genauen Wasserlauf-Geländemodells beschrieben. Die Prozesskette beginnt bei der Qualitätskontrolle und umfasst weiters die Ableitung hydraulisch relevanter Geländekanten, die Trennung der ALS-Punktwolke in Wasser-, Boden- und Nicht-Bodenpunkte sowie die Interpolation qualitativ hochwertiger digitaler Geländemodelle (DGM). Neben dem DGM sind weiters Gebäude- und Vegetationslayer für Folgeanwendungen von Interesse. Im zweiten Teil wird die Aufbereitung dichter ALS-basierter Topographiedaten für die anschließende hydrologische bzw. hydraulische Modellierung behandelt. Eine qualitativ hochwertige Ausdünnung der DGM-Daten, bei der typischerweise Reduktionsraten bis zu $99 \%$ erreicht werden, ist dabei für die erfolgreiche Anwendung in nachfolgenden Simulationen von großer Bedeutung. Abschließend wird eine geometrische Herangehensweise zur Generierung von Rechengittern beschrieben.

Topographic data from airborne laser scanning as a basis for hydrology and water resources management

Institut für Photogrammetrie und Fernerkundung Technische Universität Wien

2 Christian Doppler Labor für „Räumliche Daten aus Laserscanning und Fernerkundung" am Institut für Photogrammetrie und Fernerkundung, Technische Universität Wien
Summary: Over the past 15 years, airborne laser scanning (ALS) has revolutionised topographical surveying by affording an unprecedented data density of several points per square metre as well as an accuracy of levels better than $150 \mathrm{~mm}$. Topographical records from laser scanning as a precise basis of geometrical data have become an indispensable tool in danger-zone planning, flood-plain mapping and questions of water biology.

This article outlines the range of applications of ALS data in the practice of water resources management. A first part describes the construction of a detailed topographical model of a water-course. The process chain begins with quality control, followed by the derivation of terrain edges of hydraulic relevance, classification of the ALS point cloud as water, ground and nonground points as well as interpolation of high-quality digital terrain models (DTM). Also of interest are building and vegetation layers for follow-up uses. Part two deals with the preparation of ALS-based topographical data for the purpose of hydrological or hydraulic modelling. This involves the need for high-quality thinning of the DTM data, typically reaching reduction rates of up to $99 \%$, to permit successful use in subsequent simulations. The article winds up by describing a geometrical approach to the generation of computational grids.

\section{Einleitung}

Die extremen Hochwasserereignisse der vergangenen Jahre haben die Themen Gefahrenzonenplanung, Hochwasserschutz und -simulation in den Blickpunkt des medialen Interesses gerückt. Das Instrument zur Simulation von Strömungsvorgängen ist die hydraulisch-numerische (HN) Modellierung. Die wesentlichen Modell-Einflussgrößen sind die Fließwiderstände sowie die Topographie des Flusslaufes und der Überschwemmungsgebiete. Für die topographische Geländeaufnahme

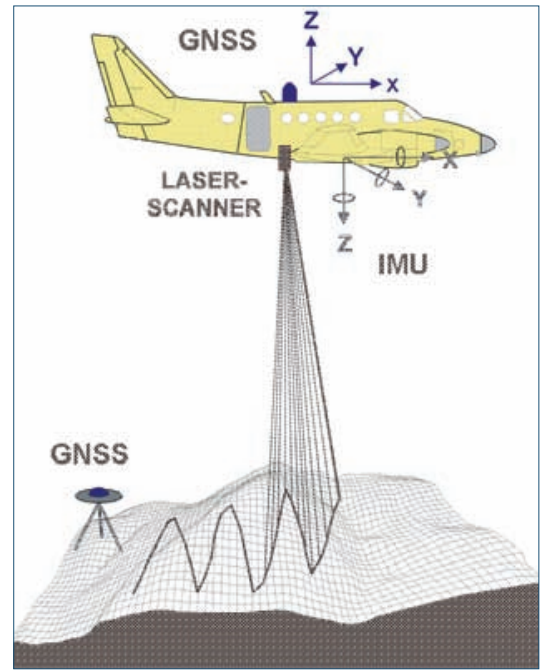

Abb. 1: Prinzipskizze des Airborne Laserscannings

im Allgemeinen und die Vermessung von Fließgewässern im Speziellen hat Airborne Laserscanning (ALS) traditionelle terrestrische oder photogrammetrische Messmethoden weitgehend abgelöst. Die wesentlichen Vorteile von ALS sind hoher Grad an Automation für flächendeckende Datenerfassung, bislang unerreichte Messpunktdichte von mehreren Punkten pro $\mathrm{m}^{2}$ und Höhengenauigkeit von ca. $10 \mathrm{~cm}$. Dadurch ist es möglich, topographische Detailstrukturen weitgehend automatisch und präzise zu erfassen. Dies gilt insbesondere für abflussrelevante Objekte wie Entwässerungsgräben, Dämme, Deiche, Böschungen von Verkehrswegen und dergleichen. Gegenüber bild-basierten Aufnahmetechniken besteht der Vorteil darin, dass das Laserlicht in der Lage ist, durch kleine Öffnungen in der Vegetationsdecke bis zum Boden zu dringen. Durch die höhere Dichte an Bodenpunkten kann das digitale Geländemodell (DGM) mit größerer Genauigkeit und Zuverlässigkeit bestimmt werden.

Das Ergebnis einer ALS Kampagne ist eine unstrukturierte Punktwolke. Damit daraus ein DGM des Wasserlaufes (DGM-W) abgeleitet werden kann, sind eine Reihe von Prozessierungsschritten zu 


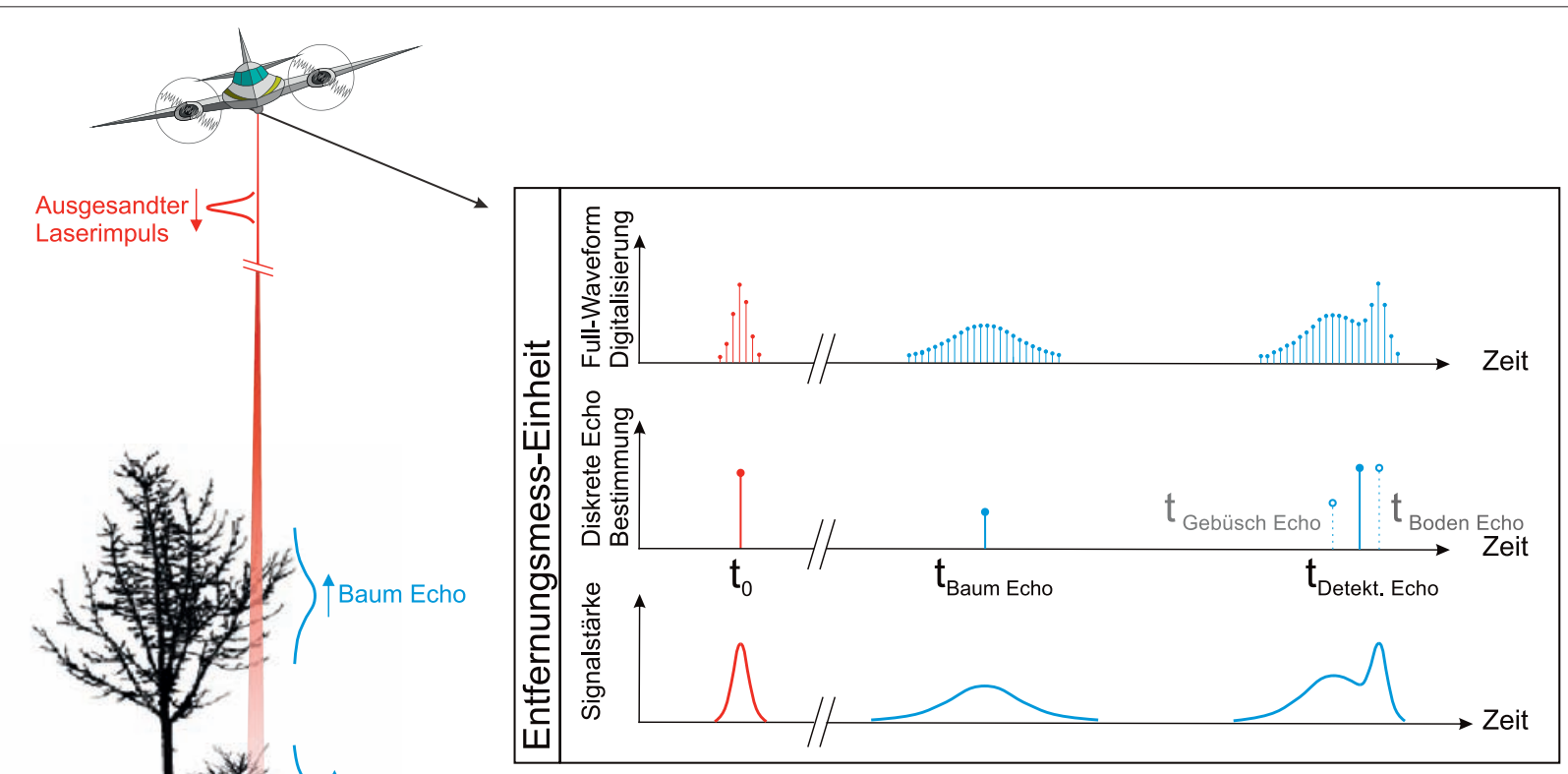

Abb. 2: Diskrete Echobestimmung vs. Full-Waveform-Digitalisierung, Prinzipskizze

durchlaufen, angefangen bei der Qualitätskontrolle der Punktwolke, über die Klassifizierung in Wasser-, Boden- und Nicht-Bodenpunkte, die Ableitung von Geländekanten bis hin zur eigentlichen DGM-Interpolation aus ALS- und Gewässerbettdaten. Darüber hinaus stellt auch die enorme Datenmenge, welche moderne ALS Sensoren liefern, ein Problem für die Weiterverarbeitung in hydrologischen oder hydraulischen Modellen dar. Geeignete Strategien zur qualitativ hochwertigen Ausdünnung und Aufbereitung der Daten sind daher erforderlich, damit das volle Potenzial der ALS Daten für die eingangs erwähnten wasserwirtschaftlichen Fragestellungen ausgeschöpft werden kann.

Das Institut für Photogrammetrie und Fernerkundung (I.P.F.) der TU Wien beschäftigt sich seit mehr als 15 Jahren intensiv mit dem Thema ALS und der Anwendung von ALS in Hydrologie und Hydraulik. In diesem Lichte gibt der vorliegende Artikel einen Überblick über Messprinzip (Abschnitt 2), Aufbau eines Wasserlauf-Geländemodells (Abschnitt 3) und Aufbereitung der DGM-Daten für die Weiterverarbeitung (Abschnitt 4).

\section{Grundlagen}

\subsection{ALS Messprinzip}

Eine auf einer fliegenden Plattform (Flugzeug, Helikopter, etc.) montierte Laserquelle sendet fortlaufend sehr kurze La- serimpulse aus. Der Laserstrahl interagiert mit der Oberfläche, das Signal wird dabei teilweise absorbiert bzw. reflektiert und das rückgestreute Echo von einer Empfangseinheit detektiert. Aus der Signallaufzeit kann dann die räumliche Distanz ermittelt werden. Beim ALS handelt es sich um ein Multisensor-System, bestehend aus einer Satelliten-Navigationseinheit (GNSS - Global Navigation Satellite System) für die absolute Positionierung, einer inertialen Messeinheit (IMU - Inertial Measurement Unit) zur Erfassung der Drehwinkel (Raumstellung) des Messsystems und dem eigentlichen Laserscanner ( $A b b$. 1). Die Datenerfassung erfolgt streifenweise, wobei die Abweichungen zwischen überlappenden Streifen zur Qualitätsdokumentation und ggf. Fein-Georeferenzierung der Flugstreifen herangezogen werden können. Abhängig von der Geländeform und der Streifenüberlappung, bewirken die Bewegung des Messsystems in Flugrichtung und die Querablenkung des Laserstrahls im Laserscanner durch schwingende oder oszillierende Spiegel ein unregelmäßiges Punktmuster am Boden,. Die erzielbare Punktdichte hängt im Wesentlichen von der Flughöhe und der Laserimpuls-Wiederholfrequenz (pulse repetition rate) ab. Letztere liegt heutzutage bei $100-200 \mathrm{kHz}$ und es wird typischerweise eine Punktdichte von 1-20 Punkten $/ \mathrm{m}^{2}$ erreicht. Die Reflexionsbzw. Absorptionseigenschaften über Wasserflächen hängen wesentlich von der eingesetzten Wellenlänge des Lasersignals ab. Bei den kommerziell verfügbaren ALS Sensoren werden vornehmlich Laser im nahen Infrarotbereich $(\lambda=800$ $1550 \mathrm{~nm}$ ) eingesetzt, wobei die Absorption über Wasser mit zunehmender Wellenlänge zunimmt. In Tabelle 1 sind die technischen Daten typischer ALS-Systeme zusammengefasst.

\section{TABELLE 1}

Technische Daten typischer ALS Systeme

\begin{tabular}{ll}
\hline Spezifikation & Typischer Wert \\
\hline Wellenlänge & 0,9 and $1.5 \mu \mathrm{m}$ \\
\hline Impulsdauer & $5-15 \mathrm{~ns}$ \\
\hline Strahldivergenz & $0.2-2 \mathrm{mrad}$ \\
\hline Impuls Wiederholrate & $1-200 \mathrm{kHz}$ \\
\hline Gesichtsfeld & $14-75^{\circ}$ \\
\hline Scanrate & $25-650 \mathrm{~Hz}$ \\
\hline Scanmuster & $\begin{array}{l}\text { Zickzack, parallel, } \\
\text { elliptisch, sinusoidal }\end{array}$ \\
\hline Grundfläche (footprint) & $0.25-2 \mathrm{~m}$ \\
\hline Mehrfach-Echos & $\begin{array}{l}2-8 \text { oder Full-wave- } \\
\text { form }\end{array}$ \\
\hline Intensität & Ja \\
\hline Flughöhe & $300-3000 \mathrm{~m}$ \\
\hline GPS Frequenz & $1-2 \mathrm{~Hz}$ \\
\hline INS Frequenz & $128-256 \mathrm{~Hz}$ \\
\hline Genauigkeit (Höhe) & $0.10 \mathrm{~m}$ \\
\hline Genauigkeit (Lage) & $0.10-1 \mathrm{~m}$ \\
\hline
\end{tabular}


Während traditionelle Systeme diskrete Echos (meist: erstes und letztes Echo) liefern, zeichnen moderne Sensoren die gesamte Echo-Wellenform auf (Full Waveform Laserscanning). Der Vorteil liegt darin, dass im post-processing für jedes Echo neben den 3D-Punktkoordinaten $(\mathrm{X}, \mathrm{Y}, \mathrm{Z})$ zusätzliche Attribute wie Amplitude und Echoweite abgeleitet werden können, welche eine verbesserte DGM Bestimmung ermöglichen. In Abbildung 2 ist der Unterschied zwischen diskreter Echodetektion bzw. Full Waveform (FWF) Laserscanning dargestellt. Als weiterführende Literatur sei auf Wehr u. Lohr (1999), Kraus (2002) und Wagner et al. (2006) verwiesen.

\subsection{Qualitätskontrolle der ALS-Punktwolke}

An ALS Geländemodelle als Geometriegrundlage für hydraulische Modellierungen werden sehr hohe Ansprüche hinsichtlich der Höhengenauigkeitgestellt $(<10 \mathrm{~cm})$. Um diese Anforderungen sicherstellen zu können, ist eine Qualitätskontrolle der ALSPunktwolke erforderlich. Die wichtigsten Qualitätsparameter sind dabei die Punktdichte und die geometrische Genauigkeit. Zur Überprüfung der Punktdichte können Punktdichtekarten aller letzten LaserEchos herangezogen werden (Karel et al. 2006). Es handelt sich dabei um Rasterbilder, die in jedem Pixel durch Ampelfarben (Grün-Gelb-Rot) angeben, ob für den betrachteten Bereich die geforderte Punktdichte eingehalten ist. Da es sich bei der Punktdichte um ein statistisches Maß handelt, wird man die Raster-Auflösung so wählen, dass eine Rasterzelle (in Bereichen mit der erwarteten Punktdichte) zumindest 20-25 Punkte enthält. Ausschlaggebend für die Genauigkeit des DGM ist nicht die Punktdichte aller, sondern nur der als Bodenpunkte klassifizierten Echos. In Abbildung 3 ist zu erkennen, dass die angestrebte Punktdichte von 2 Punkten $/ \mathrm{m}^{2}$ (abgesehen von den Wasserflächen) für die letzten Echos erreicht wurde (a), dass dies durch die Filterung von Vegetation für die als Bodenpunkte klassifizierten Echos entlang der Straßenböschungen aber nicht der Fall ist.

Die geometrische Genauigkeit der ALS Punktwolke wird neben der Sensorkalibrierung vor allem durch die Qualität der Georeferenzierung bestimmt. Zur Überprüfung der relativen Passgenauigkeit der Flugstreifen wird zunächst für jeden Streifen ein Digitales Oberflächenmodell (DOM) berechnet. Anschließend

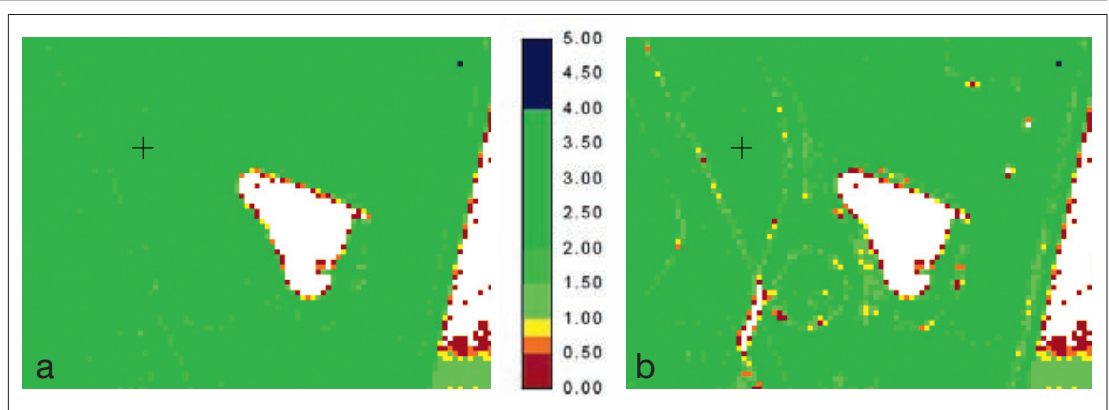

Abb. 3: Punktdichtekarte, (a) alle letzten Echos, (b) als Bodenpunkte klassifizierte Echos, angestrebte Punktdichte (=grün): 2 Punkte/m², Pixelgröße $5 \times 5 \mathrm{~m}^{2}$

werden aus den jeweils überlappenden Modellen Streifen-Differenzmodelle abgeleitet. Diese werden mittels eines Rauigkeitslayers maskiert, da ein Vergleich nur an offenen und glatten Flächen zulässig ist, und farbkodiert dargestellt (Ressl et al. 2008). Die Farbpalette wird dabei so gewählt, dass kleine Abweichungen durch blasse, große aber durch kräftige, auffällige Farben repräsentiert sind. Die Visualisierungen der Streifendifferenzen können nicht nur einen systematischen Höhenversatz der Streifen, sondern oft auch Lageverschiebungen anzeigen. Kräftige Farbtöne entlang geneigter Flächen (Böschungen, Dächer, etc.) sind diesbezügliche Indikatoren (vgl. Abb. 4a). Mittels Least Squares Matching können an korrespondierenden Flächenstücken auch 3DVerschiebungen ermittelt werden. Auf Basis dieser Information kann schließlich eine qualifizierte Aussage getroffen werden, ob eine zusätzliche Fein-Georeferenzierung der Punktwolke mittels Streifenausgleichung (Ressl et al. 2009; Kager 2004) erforderlich ist.

\section{Aufbau eines Wasserlauf- Geländemodells}

Das Wasserlauf-DGM stellt die geometrische Grundlage für die hydraulische Modellierung dar. Es bildet den überflutungsrelevanten Bereich eines Flusslaufes einschließlich des Gewässerbetts ab (Brockmann und Mandlburger 2001). Neben dem Gewässerbett, der Uferböschung und dem Flussvorland sind im DGM-W auch alle abflusshindernden Bauwerke, wie Brücken oder Wehre, zu integrieren. Das DGM-W wird daher aus Daten unterschiedlicher Erfassungsmethoden aufgebaut. Eine bewährte Methode zum Aufbau des DGM-W aus ALS Daten zur Beschreibung des Flussvorlandes und Echolot Querprofilen zur Modellierung der Gewässersohle wird im Folgenden überblicksartig vorgestellt.

\subsection{Automatische Modellierung} hydraulisch relevanter 3D-Geländekanten

Geländekanten repräsentieren Unstetigkeitsstellen in der Geländeneigung und sind deswegen für die hydraulische Modellierung von besonderer Bedeutung. Sie markieren oftmals die Begrenzung von Rauigkeitszonen (z.B. Uferböschungsoberkante) und hydraulisch relevanten Objekten (Dämme, Deiche, Gräben, etc.). Die Einbeziehung von Geländekanten ins DGM ermöglicht eine präzisere Beschreibung der Topographie aber auch die Reduktion der DGM-Datenmenge. Geländekanten sind darüber hinaus auch für das hydraulische Berechnungsnetz essentiell.

Die meisten Ansätze in der Literatur sind rasterbasiert (z. B. Brügelmann 2000). Die Rasterisierung setzt allerdings eine vorherige Klassifikation der Bodenpunkte voraus, was i. A. zu einer Glättung gerade im Bereich von Geländekanten führt. Am I.P.F. wurde daher in den vergangenen Jahren eine Methode zur automatischen Modellierung von Geländekanten auf Basis der ALS-Punktwolke entwickelt (Briese 2004; Briese et al. 2009). Der Ansatz besteht im Wesentlichen aus zwei Teilen: (i) Detektion von potentiellen Startsegmenten und (ii) Modellierung von 3D-Geländekanten auf Basis der Startsegmente aus (i). Im Detektions-Schritt wird in jedem Punkt der ALS-Punktwolke aus den $\mathrm{k}$ nächsten Nachbarn eine Fläche zweiter Ordnung (Quadrik) geschätzt, woraus die maximale und minimale lokale Krümmung $\left(\kappa_{\max }, \kappa_{\min }\right)$ abgeleitet werden. Mittels geeigneter Schwellwerte für die maximale Krümmung $\left(\kappa_{\max }\right)$ und das Krümmungsverhältnis $\left(\kappa_{\max } / \kappa_{\min }\right)$ können Startsegmente ermittelt werden. Diese dienen im nachfolgenden Modellierungsschritt als Näherungen. Die Modellierung der Kanten selbst erfolgt dabei Patchweise durch den Verschnitt von Ebenenpaaren mittels robuster Ausgleichung 


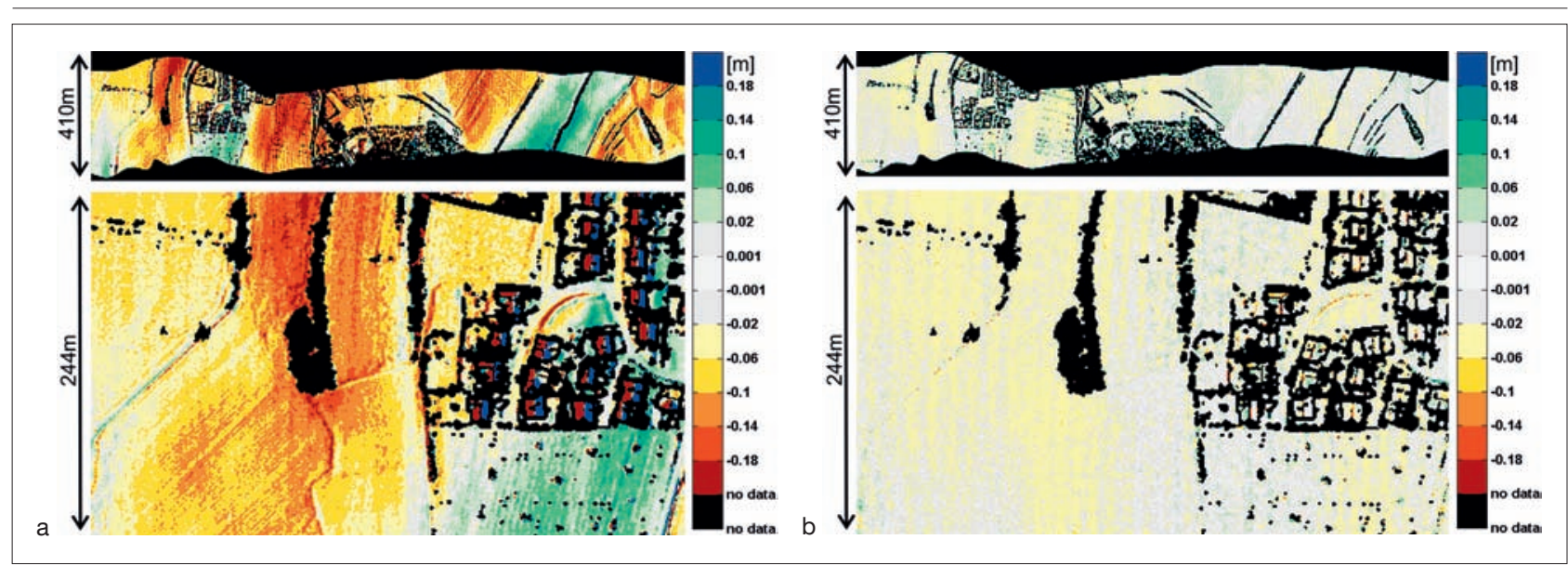

Abb. 4: Farbkodierte Streifendifferenzen für die originale Georeferenzierung (a) und für die verbesserte Georeferenzierung nach einer Streifenausgleichung (b)

nach kleinsten Quadraten, wobei der robuste Ansatz eine automatische Elimination des Vegetationseinflusses erlaubt. Die Schnittgerade der beiden Ebenen beschreibt eine Tangente an die Geländekante, und in der Mitte des Flächen-Patches wird dann ein repräsentativer Kantenpunkt bestimmt. Anschließend wird sowohl in die Vor- als auch Rückrichtung extrapoliert und der Prozess solange wiederholt, bis das Abbruchskriterium erreicht ist (z. B. Schnittwinkel der Ebenen kleiner $10^{\circ}$ ). Abbildung 5 zeigt für ein praktisches Beispiel die aus der ALS-Punktwolke automatisch generierten Startsegmente und 3D-Geländekanten.

\subsection{Filterung der ALS-Punktwolke}

Im Bereich des Flussvorlandes wird das DGM-W aus den Bodenpunkten des ALSDatensatzes aufgebaut. Dazu ist eine Filterung der ALS-Punktwolke in Boden- und Nicht-Bodenpunkte erforderlich. In der Literatur wurden dazu eine Reihe von Verfahren vorgeschlagen, die grundsätzlich alle auf geometrischen Kriterien, i. A. den Höhenverhältnissen benachbarter Punkte, beruhen. In diese Kategorie gehört auch die am I.P.F. entwickelte Methode der robusten hierarchischen Interpolation (Kraus und Pfeifer 1998; Pfeifer et al. 2001). Einen Vergleich der gängigsten Filtermethoden enthält (Sithole und Vosselman 2004). Eine Verbesserung des Filterergebnisses lässt sich durch Einbeziehung der bereits davor explizit modellierten Geländekanten erreichen. Weiteres Potenzial besteht durch die Berücksichtigung von zusätzlichen Echo-Attributen, die aus der Analyse der gesamten Wellenform des reflektierten Lasersignals gewonnen werden können. Die Echoweite etwa ist ein Maß für die Ausdehnung eines Objektes innerhalb des Laserstrahls (z. B. Höhenverteilung in der Krone) und kann daher zur Vor-Klassifizierung der ALS-Punktwolke herangezogen werden (Briese et al. 2007). Für die robuste Interpolation kann die Echoweite darüber hinaus zur Bestimmung von a-priori-Punktgewichten herangezogen werden (Mandlburger et al. 2007). Dadurch wird die Zuverlässigkeit der Filterung vor allem in dem für hydraulische Anwendungen sensiblen Bereich der niedrigen Vegetation (Echoaufwei- tung) weiter gesteigert und die Qualität des abgeleiteten DGM verbessert. Abbildung 6 illustriert das Verbesserungspotenzial der ALS-Filterung bei zusätzlicher Verwendung der Echoweite für die Klassifikation in Boden- und Nicht-Bodenpunkte.

\subsection{Abgrenzung von Wasserflächen}

Die Trennung von Punkten des aquatischen und trockenen Bereiches erfolgt auf Basis der Wasser-Land-Grenzlinie (WLG). Im Folgenden sind zwei Verfahren beschrieben: Eine rein geometrische Lösung auf Basis der ALS-Punktwolke und Querprofildaten (Brockmann und Mandlburger 2001) und eine Methode, welche die geometrischen und radiometrischen Eigenschaften der ALS-Punktwolke nutzt (Höfle et al. 2009). Das erste Verfahren beruht auf einem Verschnitt eines DGM (Stufe 1) bzw. DGM-W (Stufe 2) mit einem digitalen Modell der Wasseroberfläche (DWM), wobei das DWM aus den gelegentlichen Reflexionen des Lasersignals an der Wasseroberfläche aufgebaut werden kann. Der Verschnitt wird durch die Berechnung eines Differenzmodells aus
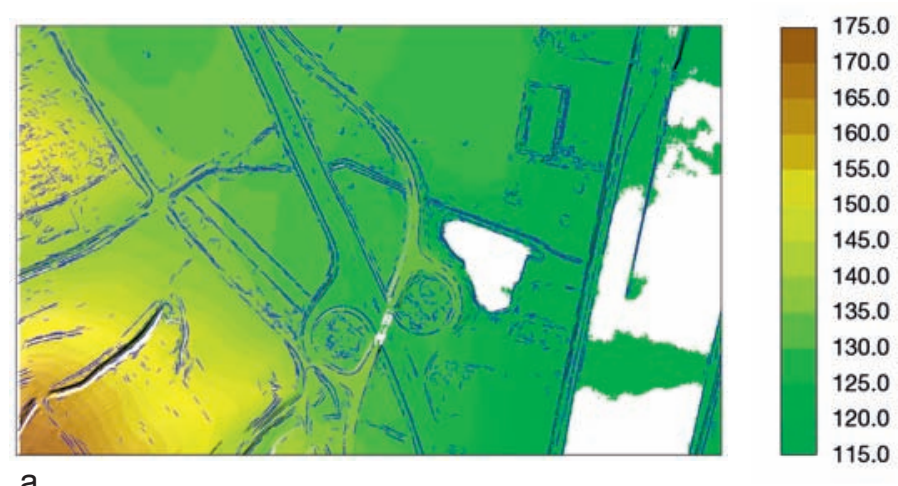

a

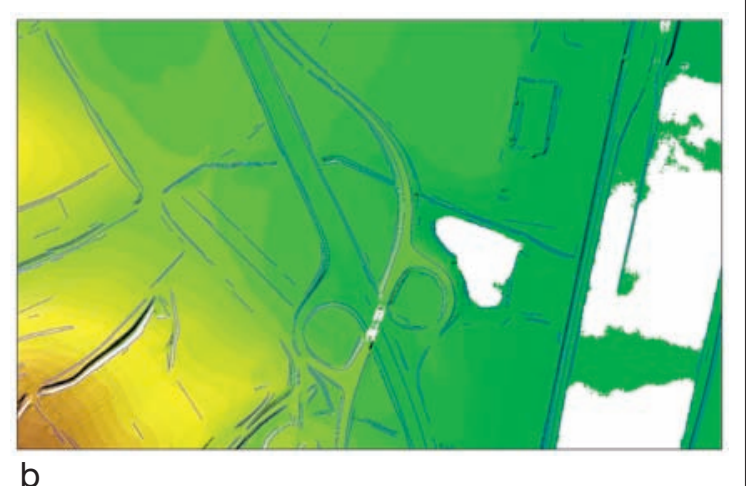

Abb. 5: Automatisch detektierte Startsegmente (a) und automatisch modellierte 3DGeländekanten (b) jeweils blau, Rastergrafik: DGM-Schummerung überlagert mit Höhenkodierung 


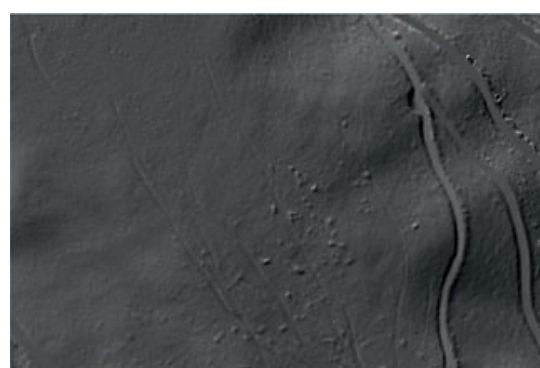

a

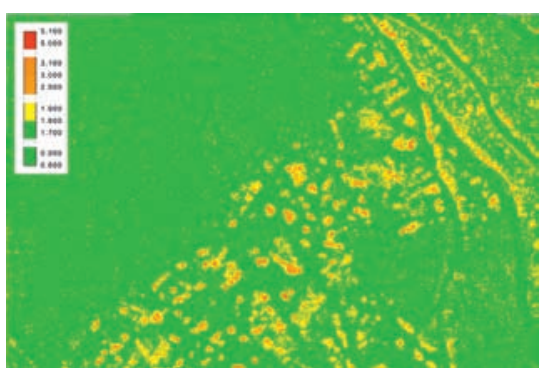

b

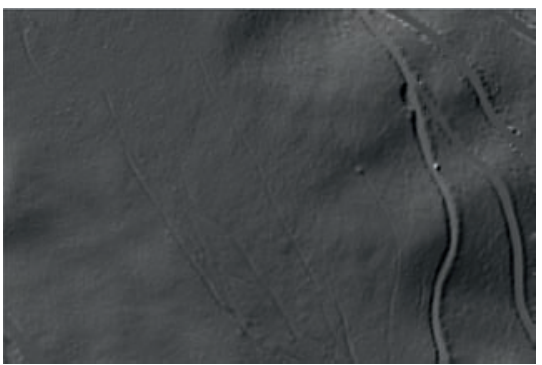

C

Abb. 6: (a) Schummerung eines DGM , Klassifizierung der ALS-Punktwolke mittels Standard-ALS-Filterung, (b) farbkodierte Darstellung der Echoweite, grün = kleine Echoweite $=$ Bodenpunkte, gelb-rot $=$ mittel-große Echoweite $=$ Hinweis für Vegetationspunkte, (c) verbessertes DGM durch Einbeziehung grün $=$ kleine Echoweite $=$ Bodenpunkte, gelb-rot $=$ mittel-große Echoweite $=$ Hinweis für Vegetat
der Echoweite für die Filterung der ALS-Punktwolke (niedrige Vegetation erfolgreich eliminiert)

DGM und DWM realisiert, aus welchem die WLG als Null-Isolinie extrahiert wird. Die Prinzipskizze in Abbildung 7 veranschaulicht die Zusammenhänge.

In einer ersten Stufe wird zunächst ein DGM aus allen ALS-Bodenpunkten bzw. Reflexionen an der Wasseroberfläche aufgebaut und mit dem DWM verschnitten. Daraus ergibt sich eine vorläufige WLG, die aufgrund schleifender Schnitte schlecht definiert ist. Die ALS-Punkte innerhalb der vorläufigen WLG werden durch Punkte des Gewässerbettes ersetzt, und anschließend (Stufe 2) kann aus den verbleibenden ALSPunkten und den Gewässerbettdaten ein vorläufiges DGM-W berechnet werden. Die endgültige Wasser-Land-Grenze gewinn man schließlich aus dem neu berechneten Differenzmodell zwischen vorläufigem DGM-W und DWM.

Beim Ansatz von Höfle et al. (2009) wird der Umstand ausgenutzt, dass das Lasersignal auf der Wasseroberfläche meist stark absorbiert wird und daher eine geringe Amplitude bzw. in der Nadirrichtung durch den „Spiegel-Effekt“ eine extrem hohe Amplitude aufweist. Die wegen der Absorption des Lasersignals fehlenden Echos (dropouts) werden dabei als QuasiPunkte mit Null-Amplitude eingeführt. Ferner zeichnen sich benachbarte Wasserpunkte durch geringe Höhenvariation aus. Mit dieser Information wird eine Seeded Region Growing Segmentierung durchge- führt. Die endgültige Trennung in Wasserund Nicht-Wasserpunkte erfolgt durch eine objektbasierte Klassifikation der Segmente, wobei Wasserpunkte die PseudoHöhe $\mathrm{z}=0$ zugewiesen bekommen und Nicht-Wasserpunkte $\mathrm{z}=1$. Die WLG ergibt sich dann aus dem Pseudo-Höhenmodell mit der Isolinie 0.5. Höfle et al. (2009) berichten, dass der Ansatz auf Fließgewässer unterschiedlicher Breite und Neigung anwendbar ist. Die Klassifikationsgenauigkeit beträgt $>97 \%$ und die planimetrische Abweichung (r.m.s.) gegenüber GPS-Referenzmessungen $\pm 0.45 \mathrm{~m}$. Ein praktisches Ergebnis der Wasserflächendetektion mit dieser Methode am Inn zeigt die Abbildung 8. Die Wasseroberfläche des Inns und des Baggersees konnten erfolgreich vollautomatisch abgegrenzt werden. Die Brücke sowie einzelne Sand- und Schotterbänke sind deutlich zu erkennen (markiert mit Pfeilen).

\subsection{Verdichtung von Gewässerbett- Querprofilen}

Wie bereits erwähnt, kommen in kommerziell verfügbaren ALS Systemen Laser mit Wellenlängen im nahen Infrarotbereich zum Einsatz. Bei dieser Wellenlänge wird der Laserimpuls an der Wasseroberfläche absorbiert bzw. reflektiert und die Gewässersohle kann damit nicht bestimmt werden. Es existieren aber auch Lasersysteme, die für die Erfassung von Gewässertiefen ausgelegt sind (Laser Bathymetrie). Dabei werden gleichzeitig zwei verschiedene Wellenlängen verwendet: Nahes Infrarot $(\lambda=1064 \mathrm{~nm})$ für die Reflexion an der Wasseroberfläche und grün-blaues Laserlich $(\lambda=532 \mathrm{~nm})$, welches Wasser durchdring und an der Gewässersohle reflektiert wird. Für den Einsatz bei den fest- und schwebstoffreichen Flüssen unserer Breiten is die Laser Bathymetrie allerdings nicht geeignet, da die Gewässertrübheit den limitierenden Faktor darstellt. Die Beschreibung der Gewässerbett-Geometrie erfolgt daher meist auf Basis von tachymetrisch oder mittels Echolot vermessenen Querprofilen. Damit die Punkte des Gewässerbettes gemeinsam mit den ALS-Bodenpunkten $\mathrm{zu}$ einem homogenen DGM-W interpoliert werden können, sind diese noch entsprechend aufzubereiten. Eine Profilverdichtung ist notwendig, da der Profilabstand in der Regel wesentlich höher ist als der Abstand der Punkte innerhalb eines Profils. Qualitativ hochwertige Algorithmen verwenden die gekrümmte Flussachse als Grundlage zur Homogenisierung der Punktverteilung und erlauben dadurch die Einbeziehung der Hauptfließrichtung in die Berechnung von Zwischenprofilen. Für weitere Details sei auf die Literatur verwiesen (Flanagin et al. 2007; Mandlburger und Strobelberger 2007; Vetter et al. 2008).

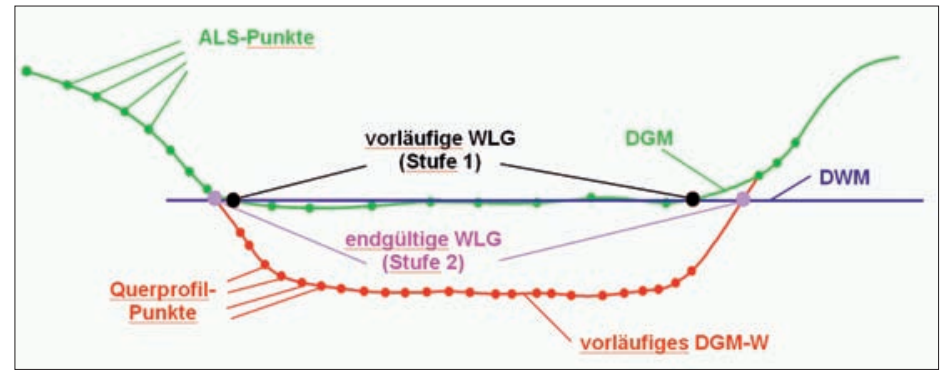

Abb. 7: Prinzipskizze zur zweistufigen Ableitung der WLG aus dem Verschnitt von DGM bzw. DGM-W und DWM

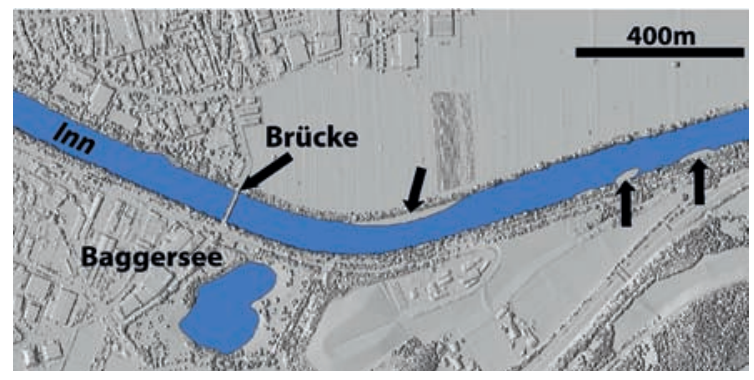

Abb. 8: Wasserflächendetektion nach Höfle et al. (2009), Inn bei Rossau/Innsbruck. 


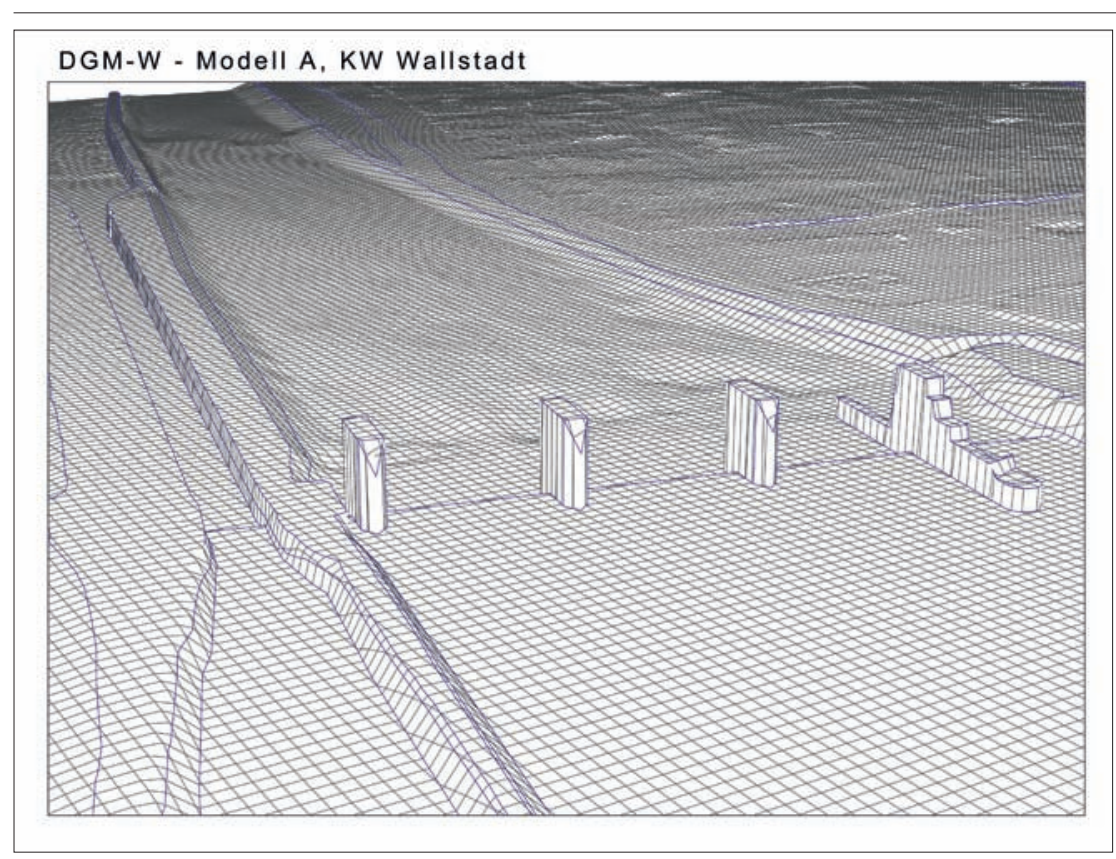

Abb. 9: Digitales Wasserlauf-Geländemodell aufgebaut aus ALS-Punkten (Vorland), Echolotdaten (Gewässerbett), automatisch modellierten Geländekanten und Bauwerksdaten, Perspektivansicht des hybriden Geländemodells (regelmäßiges 3m-Gitter (grau) + eingekettete Geländekanten (blau)), Main bei Wallstadt, Deutschland

\subsection{Interpolation des DGM-W}

Nach den in den vergangenen Abschnitten beschriebenen Vorbereitungsschritten, können die Geländekanten, die ALS-Bodenpunkte, die Gewässerbett- und etwaige Bauwerksdaten $\mathrm{zu}$ einem homogenen DGM-W interpoliert werden. Um den Einfluss zufälliger Messfehler zu minimieren empfiehlt sich dabei der Einsatz einer Interpolation mit qualifizierter Glättung. Abbildung 9 zeigt ein DGM-W in hybrider Geländemodellstruktur (regelmäßiges Gitter und eingekettete Geländekanten), welches mittels linearer Prädiktion (Kraus 2000) mit dem Programmsystem SCOP++ (I.P.F 2009) berechnet wurde.

\subsection{Weitere aus ALS abgeleitete Datensätze}

Neben dem DGM können aus der ALSPunktwolke noch weitere Datensätze abgeleitet werden, die sowohl für die hydrologische als auch hydraulische Modellierung wertvoll sind. Im Bezug auf die Vegetation kann z. B. die Eigenschaft, dass der Laserstrahl zuerst von der Baumkrone (erstes Echo) und im weiteren Verlauf vom Boden (letztes Echo) reflektiert wird, ausgenutzt werden, um Vegetationslayer zu erstellen. Dabei wird aus den ersten Echos ein DOM und aus den als Bodenpunkte klassifizierten letzten Echos ein DGM berechnet. Das Differenzmodell aus DOM und DGM wird sen (Blockmodelle), oder in Form von Rauigkeiten im hydraulischen Berechnungsnetz.

\section{Aufbereitung topographischer Daten für die hydraulische Modellierung}

Der Einsatz moderner ALS Sensoren ermöglicht den Aufbau von sehr dichten $\mathrm{Ge}$ ländemodellen. Immer mehr topographische Details können zum Preis einer ständig steigenden Datenmenge effizient erfasst werden. Ein ALS-DGM-W besteh für wenige $\mathrm{km}^{2}$ Projektgebiet typischerweise aus mehreren Millionen Punkten. Im Zuge der hydraulische Modellierung erfolgt die Lösung der komplexen physikalischen Gleichungen(Navier-Stokes'sche Differentialgleichungen) meist mittels Finiter Elemente (FE) oder Finiter Volumina (FV) Ansätze. Dies erfordert eine Abstraktion der Geländeform in diskrete Zellelemente. Auf die Datenreduktion dichter ALS-Geländemodelle und Aspekte, betreffend den Aufbau hydraulischer Berechnungsnetze, wird daher im Folgenden näher eingegangen.

\subsection{Datenreduktion dichter ALS- Geländemodelle}

Einen Überblick über vorhandene Methoden zur Vereinfachung von Flächen geben Heckbert und Garland (1997). Die gängigsten Ansätze sind statische Ausdünnung regelmäßiger Gitter, weiters Dezimierungs- und Verfeinerungstechniken. Die statische Gitterausdünnung ist weit verbreitet und einfach, liefert aber suboptimale Qualität, da häufig bedeutsame Punkte verloren gehen. Eine qualitativ hochwertigere Approximation kann durch Dezimierungs- oder Verfeinerungstechniken auf Basis allgemeiner Dreiecksvermaschung, wie der Delaunay Triangulation, erreicht werden. Dezimierungsansätze arbeiten vom Feinen ins Grobe und sind für die Datenreduktion dichter ALS-DGMe nicht geeignet, da sie eine Triangulation der gesamten Punktwolke erfordern. Die Methode der Verfeinerung (mesh refinement) hingegen startet mit einer kleinen Menge ausgewählter Startpunkte und arbeitet vom Groben ins Feine. In einem iterativen Prozess werden immer mehr Punkte eingefügt, solange bis die geforderte Höhentoleranz gegenüber der ursprünglichen Fläche eingehalten oder eine gewisse maximale Punktanzahl erreicht ist. Hohe Reduktionsraten können nur für glatte Flächen erreicht werden, bei 


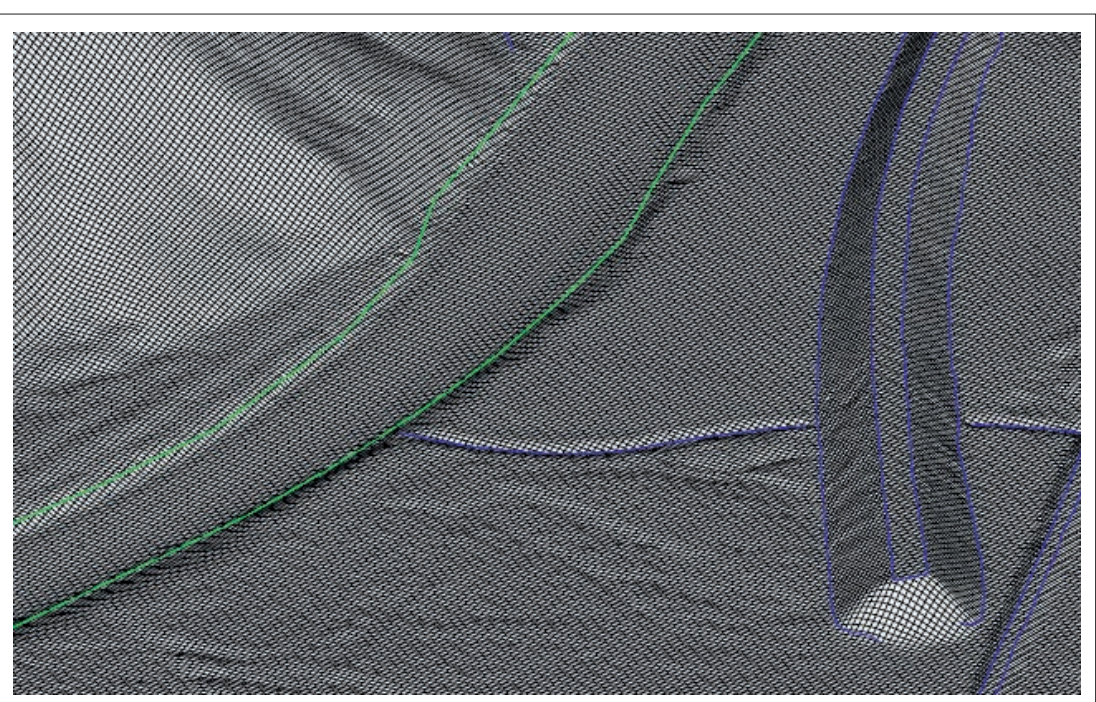

a

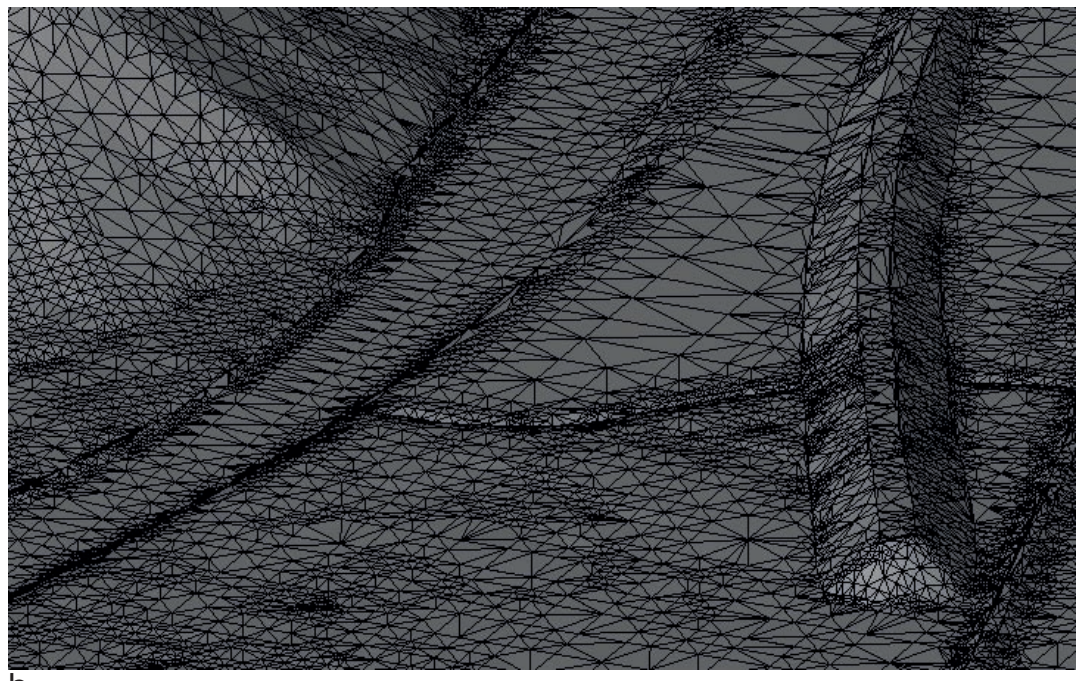

$\mathrm{b}$

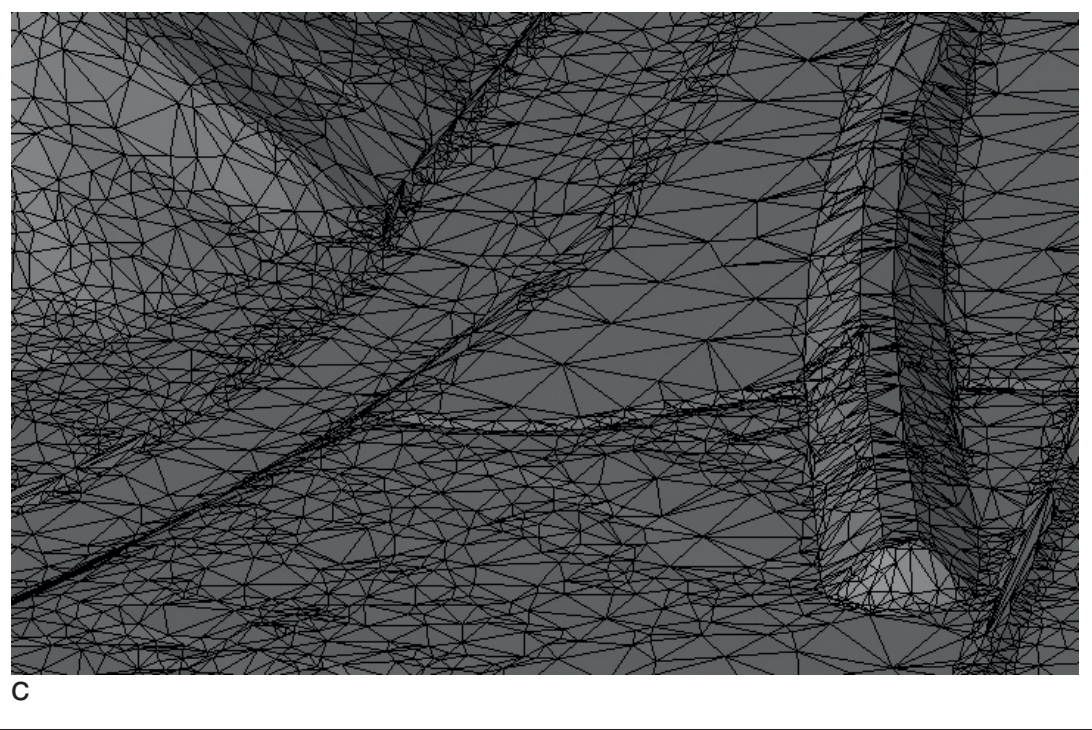

Abb. 10: Originales DGM-W in hybrider Datenstruktur, Gitterweite: $2 \mathrm{~m}$ (a) und generalisierte TINs, (b) hierarchische Unterteilung, $\Delta \mathrm{z}_{\max }=25 \mathrm{~cm}$, Reduktionsrate: $83 \%$, (c) unregelmäßige Unterteilung, $\Delta \mathrm{z}_{\max }=25 \mathrm{~cm}$, Reduktionsrate: $94 \%$ denen zufällige Messfehleranteile zuvor durch geeignete Interpolationsverfahren minimiert worden sind. Der im Folgenden vorgestellte Reduktionsansatz mittels adaptiver TIN-Verdichtung (Mandlburger 2006) setzt daher nicht auf der originalen ALS-Punktwolke auf, sondern auf einem gefilterten DGM-W in hybrider Datenstruktur, bestehend aus einem regelmäßigen Raster zur Beschreibung der glatten Geländebereiche und eingeketteter $\mathrm{Ge}$ ländekanten, welche die Unstetigkeiten im Geländeverlauf abbilden.

Die wesentlichen Parameter der DGM Datenreduktion sind eine maximal zulässige Höhentoleranz $\Delta z_{\max }$ und ein maximal zulässiger Punktabstand $\Delta \mathrm{xy}_{\max }$ Letzterer verhindert das Entstehen von Dreiecken mit zu langen Seiten bzw. zu kleinen Winkeln. Der Algorithmus startet mit einer initialen Approximation, bestehend aus den DGM-Strukturlinien und einer regelmäßigen Auswahl von Gitterpunkten (Punktabstand $=\Delta \mathrm{xy}_{\max }=\Delta 0$ ). Dieses Subset des originalen DGM-W wird mittels einer Constrained Delaunay Triangulierung vermascht, wobei die Geländekanten als Zwangskanten in das Dreiecksnetz (Triangular Irregular Network, TIN) eingefügt werden. Anschließend wird jede $\Delta 0$-Kachel durch iteratives Einfügen weiterer Gitterpunkte solange verfeinert, bis die maximale Höhentoleranz $\Delta z_{\max }$ eingehalten ist.

Das Einfügen zusätzlicher Punkte kann entweder hierarchisch oder unregelmäßig erfolgen. Im Falle der hierarchischen Unterteilung dient ein rechteckiges Gitter als initiale Flächenapproximation. Jede Zelle dieses groben Rechtecksgitters wird in vier Quadranten unterteilt, sofern ein einziger DGM-Gitterpunkt die erlaubte Höhentoleranz überschreitet. Das führt zu einer Quad-Tree artigen Datenverteilung. Im Gegensatz dazu wird bei der unregelmäßigen Unterteilung eine Grundstruktur aus annähernd gleichseitigen Dreiecken verwendet. Dies ist vor allem für FE-Gitter vorteilhaft, da die Verbindungslinien der Zellmittelpunkte (=Rechenrichtung) mit der Zellberandung stets einen rechten Winkel einschließen. In einem Durchgang werden jeweils die Punkte mit der größten positiven und negativen Abweichung ins TIN eingefügt (parallel greedy insertion), wobei der iterative Prozess des Punkteinfügens und Neuberechnens der Abweichungsbeträge derart optimiert ist, dass nur die von Änderungen betroffenen TINZellen in Betracht gezogen werden. Höhere Kompressionsraten (von bis zu 99\% 


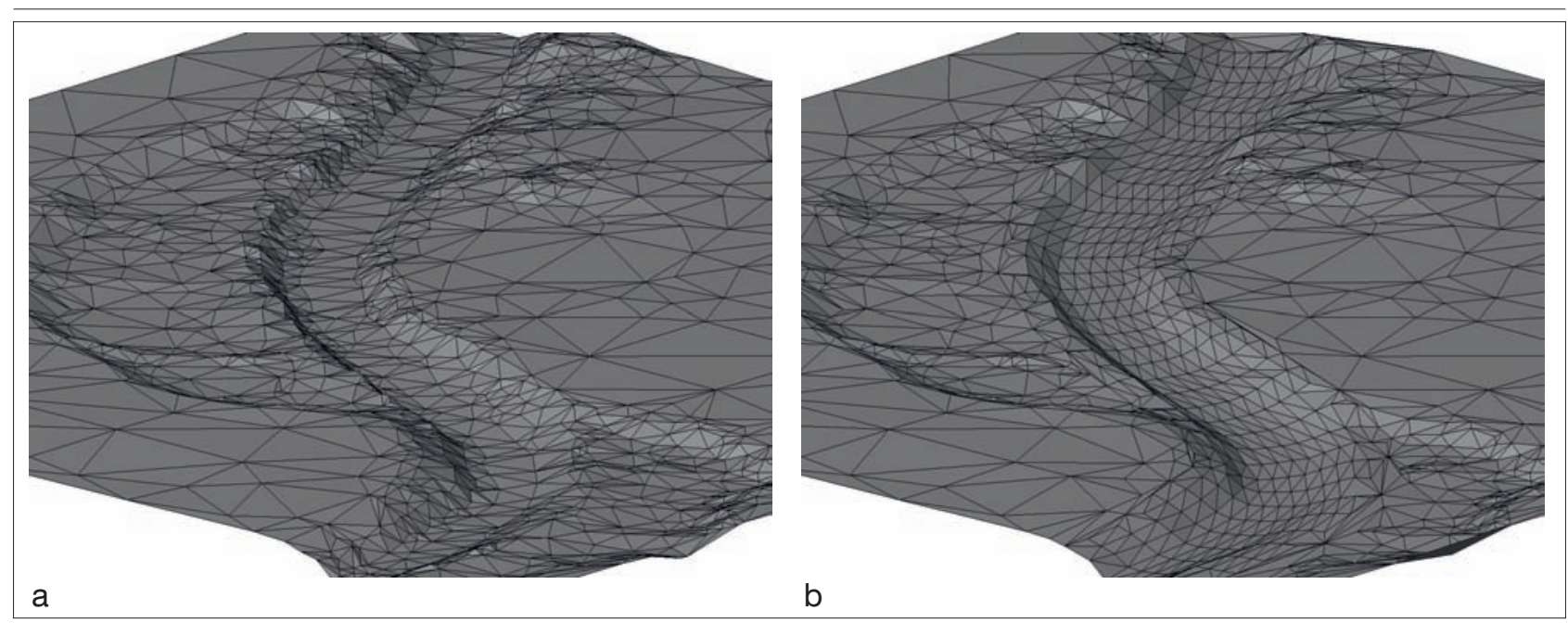

Abb. 11: Datenaufbereitung im Bereich des Gewässerbettes, (a) Adaptives TIN (unstrukturiertes Gitter), (b) profilorientierte Punktanordnung (hybrides Gitter)

bei flachem Gelände) können mit der unregelmäßigen Unterteilung erzielt werden, wogegen die hierarchische Unterteilung zu einer homogeneren Punktverteilung führt. Mit den Begriffen der Hydrodynamik ausgedrückt, führt die unregelmäßige Unterteilung zu einem unstrukturierten Gitter und die hierarchische zu einem adaptiven kartesischen Gitter. Der beschriebene Algorithmus ist flexibel hinsichtlich des Kriteriums, wann ein Punkt neu eingefügt werden soll. Diese Entscheidung kann entweder durch Analyse der lokalen Flächenneigung und -krümmung oder durch direkte Berechnung der Höhendifferenz zwischen DGM$\mathrm{W}$ und approximierendem TIN erfolgen. Abbildung 10 zeigt eine Gegenüberstellung eines DGM-W und der reduzierten TIN-Varianten mit hierarchischer und unregelmäßiger Unterteilung.

\subsection{Netzgenerierung: eine geometrische Herangehensweise}

Der große Vorteil von ALS Geländemodellen besteht darin, dass topographische Details im DGM-W abgebildet werden, welche oftmals dafürverantwortlich sind, dass es zu Überströmungen kommt oder diese gerade verhindert werden. Um qualitativ hochwertige Berechnungsnetze für die hydraulische Modellierung zu erhalten, müssen allerdings neben rein geometrischen auch physikalische Parameter berücksichtigt werden. Die wichtigsten Qualitätsparameter für Netze hydraulischer Modelle sind nach Ferziger und Periz (2002): (i) Winkelkriterium, (ii) Seitenverhältnis und (iii) Ausdehnungsverhältnis. Das Seitenverhältnis bezeichnet den Quotien- ten aus längster und kürzester Seite eines Zellelementes, und das Ausdehnungsverhältnis ist ein Maß für den Flächenunterschied benachbarter Zellen. Im Bezug auf das Winkelkriterium ist zu beachten, dass die Winkel innerhalb der Kanten einer Zelle nicht zu klein werden und die Zellen in Richtung der vorherrschenden Krafteinwirkung auszurichten sind. In kurzen Worten zusammengefasst: Winkeln kleiner als $10^{\circ}$ sollen vermieden und die Zellen im Bezug zur Hauptfließrichtung ausgerichtet werden. Das Seitenverhältnis sollte 10 nicht übersteigen (Optimum: <3) und das Ausdehnungsverhältnis darf nicht größer sein als 3 (Optimum: <1.2).

Um physikalisch zuverlässige Ergebnisse zu erhalten, müssen die Zellen des Berechnungsnetzes innerhalb des gesamten bei Hochwasser benetzten Umfanges parallel zur Strömungsrichtung angeordnet sein. Dies betrifft das Gewässerbett und die Uferböschung. Die Flussachse kann dabei als genügend genaue Approximation der Fließrichtung herangezogen werden. Rechteckige Zellen mit der längeren Seite in Fließrichtung und der kürzeren Seite quer dazu in einem Verhältnis von 3:1 haben sich in zahlreichen praktischen Simulationen bewährt. Jenseits der Uferböschung im Flussvorland sind die Strömungsrichtungen nicht mehr strikt parallel, daher ist eine unregelmäßige Punktanordnung, wie oben beschrieben, zulässig. Darüber hinaus kann der Überschwemmungsbereich in Zonen unterschiedlicher Gefährdung eingeteilt werden. So sollte z.B. Wohngebiet in unmittelbarer Nähe zum Fluss im Netz detaillierter abgebildet sein als weiter entfernte oder höher gelegene
Wiesen- und Ackerflächen. Das Konzept unterschiedlicher Kartierungsgenauigkeit kann im beschriebenen DGM Datenreduktionsansatz sehr einfach durch die Einführung räumlich variabler Höhentoleranzen $\left(\Delta \mathrm{z}_{\max }=\mathrm{f}(\mathrm{x}, \mathrm{y})\right)$ realisiert werden. Zur Steuerung von $\Delta z_{\max }$ können Distanzen von der Flussachse oder relative Höhenunterschiede über dem Flussniveau herangezogen werden. Gute Ergebnisse wurden allerdings auch mit einem einfachen Zonenmodell erreicht (Mandlburger, 2006), wobei den einzelnen Zonen jeweils eine konstante Höhentoleranz zugewiesen wird. Die Abgrenzung der Zonen kann aus einer Pilotstudie (z. B. 1D-Simulation) stammen oder auf der Basis von DGM Visualisierungen (Schummerung, farbkodierte Höhenkarte) oder digitalen Orthophotos abgeleitet werden. Das resultierende TIN muss jedenfalls zum Schluss hinsichtlich der Einhaltung der oben zitierten Qualitätskriterien für Berechnungsnetze hydraulischer Modelle überprüft werden Ggf. müssen zusätzliche Knoten in das Netzt eingefügt, oder überflüssige entfernt werden. In Abbildung 11 sind für einen kleinen Ausschnitt ein unstrukturiertes und ein hybriden Rechengitter einander gegenübergestellt.

\section{Zusammenfassung und Ausblick}

In diesem Artikel wurde ein Überblick gegeben, wie Topographiedaten aus Airborne Laserscanning gewonnen und für hydrologische und wasserwirtschaftliche Fragestellungen angewendet werden können. Im Vordergrund stand dabei das Digitale Wasserlauf-Geländemodell als geo- 
metrische Grundlage für die hydraulische Modellierung. Nach einer kurzen Einführung in das Messprinzip wurde die gesamte Prozessierungskette, beginnend bei der Qualitätskontrolle der ALS-Punktwolke, über die Ableitung von hydraulisch relevanten Geländekanten, die Klassifizierung in Wasser-, Boden- und Nicht-Boden, bis hin zur Interpolation eines qualitativ hochwertigen DGM erläutert. Der zweite Teil des Artikels hatte die fachgerechte Aufbereitung von ALS Geländemodellen bis hin zur Generierung des Berechnungsnetzes für die hydraulische Modellierung zum Thema.

Die Entwicklung der Datenerfassungsmethode ALS ist weder im Bereich der Gerätetechnik noch der Algorithmik abgeschlossen. Vielmehr führen neue tech- nische Möglichkeiten immer wieder zu verbesserten Algorithmen, welche in der Folge wieder neue Einsatzbereiche erschließen. Als Beispiel dafür wurde die Einführung des Full Waveform Laserscannings genannt, welches auf der einen Seite geholfen hat, bestehende Methoden, wie die Klassifizierung der ALS Punktwolke, zu verbessern und robuster zu machen, aber auf der anderen Seite auch ganz neue Anwendungen, wie die Ableitung von Rauigkeitsbeiwerten auf Punktniveau, zu ermöglichen scheint. In jedem Fall wird klar, dass die vorhandenen technischen Möglichkeiten eine stärkere interdisziplinäre Zusammenarbeit zwischen GeodätInnen und HydrologInnen bzw. HydraulikerInnen erforderlich macht. Der vorliegende Artikel möchte dazu einen bescheidenen Beitrag leisten.

\section{Danksagung}

Wir danken folgenden Institutionen für die Bereitstellung von Daten: Bundesansalt für Gewässerkunde, Koblenz ( $A b b$. 3, 4, 5 und 9), alpS - Zentrum für Naturgefahren Management, Innsbruck und Institut für Geographie, Univ. Innsbruck (Abb. 8) sowie Firma GeoConsult/Wien (Abb. 10 und 11).

Korrespondenz:

DI Dr. Gottfried Mandlburger

Institut für Photogrammetrie und Fernerkundung Gußhausstraße 27-29, 1040 Wie

el.: 58801-12235

E-Mail: gm@ipf.tuwien.ac.at

\section{LITERATUR}

Briese C (2004) Three-dimensional modelling of breaklines from airborne laser scanner data. ternational Archives of Photogrammetry and Remote Sensing, Vol.XXXV, B3, Istanbul.

Briese C, Doneus M, Pfeifer N, Melzer T (2007) Verbesserte DGM-Erstellung mittels Full-Waveform Airborne Laserscanning. Publikationen der Deutschen Gesellschaft für Photogrammetrie, Fernerkundung und Geoinformation e.V., Band 16, E. Seyfert (ed.), S. 215-222.

Briese C, Mandlburger G, Ressl C (2009) Automatic Break Line Determination for the generation of a DTM along the river Main, ISPRS Workshop Laserscanning 2009, Paris, submitted.

Brockmann H, Mandlburger G (2001) Aufbau Brock eines der Grenzoder. Publikation der Deutschen Geselschaft für Photogrammetrie und Fernerkundung, Band 10, S. 199-208.

Brügelmann R (2000) Automatic breakline detection from airborne laser range data. International Archives of Photogrammetry and Remote Sensing, XXXIII, B3, Amsterdam, S. 109-115. Dorninger P, Pfeifer N (2008) A Comprehensive Automated 3D Approach for Building Extraction, Reconstruction, and Regularization from Airborn Laser Scanning Point Clouds, Sensors, 8(11), S. 7323-7343.

Ferziger J, Peric M (2002) Computational methods for fluid dynamics. Springer Verlag, Berlin

thods

Flanagin M, Grenotton A, Ratcliff J, Shaw K B,

Sample J, Abdelguerfi M (2007) Hydraulic Splines: A Hybrid Approach to Modeling River Channel Geometries. In: Computing in Science \& Enginering, 9/05. Los Alamitos, S. 415. Heckbert P, Garland M (1997) Survey of Polygonal Surface Simplification Algorithms / School of Computer Science, Carnegie Mellon University, Pittsburgh, PA. Version: 1997

Höfle B, Vetter M, Pfeifer N, Mandlburger G, Höle B, Vetter M, Pfeifer N, Mandlburger G, borne laser scanning using signal intensity and elevation data. Earth Surface Processes and submitted

submitted
I.P.F. (2009) www.ipf.tuwien.ac.at/products/proI.P.F. (2009) wWw.ipf.tuwien.ac.at/products/pro-
ducts.html. SCOP++ Homepage, Institut für Photogrammetrie und Fernerkundung, (27.04.2009) Kager H (2004) Discrepancies Between Overlapping Laser Scanning Strips - Simultaneous Fitting of Aerial Laser Scanner Strips. Internationa Archives of Photogrammetry and Remote Sensing, XXXV, B/1, S. 555-560.

Karel W, Briese C, Pfeifer N (2006) DTM quality assessment. International Archives of Photogrammetry and Remote Sensing, XXXVI, 2, Vienna, Austria.

Kraus K, Pfeifer N (1998) Determination of terrain models in wooded areas with airborne laser scanner data. ISPRS Journal of Photogrammetry scanner data. ISPRS Journal of Photog
and Remote Sensing 53, S. 193-203.

and Remote Sensing 53, S. 193-203.
Kraus K (2000) Photogrammetrie, Band 3, TopoKraus K (2000) Photogrammetrie, Band 3, Topographische Informationssysteme. Dümmler Verlag Mandlburger G (2006) Topografische Modelle fü Anwendungen in Hydraulik und Hydrologie. Dissertation, Technische Universität Wien.

Mandlburger G, Strobelberger G (2007) Digitale Geländemodelle zur Simulation von Überschwemmungsszenarien. In: Österreichische Zeitschrift für Vermessung und Geoinformation Zeitschrift für Vermessung

Mandlburger G, Briese C, Pfeifer N (2007) ProMandlburger G, Briese C, Pfeifer N (2007) Prochallenge for DTM generation and data administration. In Proceedings of the 51th Photogrammetric Week, D. Fritsch (ed.), Heidelberg, Germany. Herbert Wichmann Verlag.

Pfeifer N, Stadler P, Briese C (2001) Derivation of digital terrain models in the SCOP++ environment. In: Proceedings of OEEPE Workshop on Airborne Lacerscanning and Interferometric SAR for Detailed Digital Terrain Models, Stockholm, Sweden.
Kraus K (2002) Laser-Scanning - ein ParadigmaWechsel in der Photogrammetrie. Vermessung, Photogrammetrie, Kulturtechnik. 10, S. 620-624. RessI C, Kager H, Mandlburger G (2008) Quality Checking Of ALS Projects Using Statistics Of Strip Differences. International Archives of PhotoStrip Differences. International Archives of Photo-
grammetry and Remote Sensing, Vol. XXXVII, S. 253-260.

RessI C, Mandlburger G, Pfeifer N (2009) Untersuchungen zur Verbesserung der Georeferenzierung von ALS-Streifen ohne Verwendung vo GNSS-IMU-Trajektoriendaten. In: Vorträge 29. Wissenschaftlich-Technische Jahrestagung der DGPF, 18, S. 365-375.

Rottensteiner F, Briese C (2002) A New Method for Building Extraction in Urban Areas from HighResolution LIDAR Data. In: International Archives Resolution LIDAR Data. In: International Archive
of Photogrammetry and Remote Sensing, Vol. XX Photogrammetry and Remote Sensing XXXIV/3A. Graz, Austria, S. 295-301
Sithole G, Vosselman G (2004) Experimental Sithole G, Vosselman G (2004) Experimental
comparison of filter algorithms for bare-Earth extraction from airborne laser scanning point clouds. ISPRS Journal of Photogrammetry and Remote Sensing, 59. (1-2), 85-101.

Vetter M, Höfle B, Mandlburger G, Rutzinger M (2008) Ableitung von Flusssohlenmodellen aus Flussquerprofilen und Integration in Airborne Laserscanning Geländemodelle mit GRASS GIS. in: serscaning Angewant Ge 20. AGIT-Symposium Salzburg, Wichmann Verlag, S. 382-391.

Wagner W, Ullrich A, Ducic V, Melzer T, Studnicka N (2006) Gaussian decomposition and calibration of a novel small-footprint full-waveform digitising airborne laser scanner. ISPRS Journal of Photogrammetry and Remote Sensing, 60/2; S. $100-112$.

Wehr A, Lohr U (1999) Airborne laser scanning an introduction and overview. ISPRS Journal of Photogrammetry and Remote Sensing, 54/2-3, S. 68-82. 